\title{
Analysis of gait recognition system developed in NI LabVIEW
}

\author{
Dániel Salánki ${ }^{1}$, Kornél Sarvajcz \\ ${ }^{1}$ University of Debrecen, Faculty of Engineering and Mechatronics, 4028 Debrecen, Hungary
}

\begin{abstract}
Nowadays, the world of biometric identifiers is improving one of the most significantly in security technology. Within the biometric identifiers, the research group is working with the gait recognition speciality. The research group realized a complex gait recognition system in NI LabVIEW, that can detect more reference points simultaneously with a universal camera and can suit a function on the detected points. Moreover, the program can compare the functions of the reference curve with the functions of the actual curve and evaluate if the two gait images are the same or not. The program can save the actual gait data and reload them. The leg/foot ratio analyser program before the gait recognition is designed to improve accuracy. The research group tested the program in different ways: walking only in socks in shorts or in trousers, walking in slippers, walking in the opposite way. These facts have affected the gait recognition's accuracy, the lowest False Rejection Rate (FRR) was in the case of walking in socks in shorts.
\end{abstract}

\section{Introduction}

In today's world, I find it important to protect our values with the mechanical and electronic tools at our disposal. As a mechatronics engineer student, I sought to find a theme that plays a decisive role in everyday life and is closely related to mechatronics. Numerous biometric identification methods exist today (e.g. face, voice, palm, iris, retina, fingerprint authentication and DNA Deoxyribonucleic Acid - analysis), but gait recognition is less common.

This is how we came up with the idea of wanting to implement a camera-based gait recognition program using the Vision module of National Instruments LabVIEW software, which is capable of real-time image processing to recognize that the gait image of a person passing by in front of the system is the same as that of the previously stored person's gait image. If this condition is met, the system gives him / her permission. The gait recognition is preceded by a foot examination that examines the leg/foot ratio of the person. If the test is passed, the actual gait recognition begins.

\section{Gait recognition - References}

People can often feel that a familiar person is already recognized far from their walk. This common experience is the idea behind the fact that district recognition systems can be used in security technology. You can easily make an image of a person's walk, even in public places, without even getting observed by that person. Walking is also influenced by several factors: footwear, soil, fatigue, current state of mind or any injury [1].

${ }^{1}$ Corresponding author: danisalanki@yahoo.com
Walking can be defined as a series of cyclic and coordinated movements that result in human displacement [1]. Gait is defined as "a manner of moving on foot; way of walking or running" in Webster's New Collegiate Dictionary. However, human gait is more than that: it is an idiosyncratic feature of a person that is determined by, among other things, an individual's weight, limb length, footwear, and posture combined with characteristic motion [2]. Compared to physiological biometric identification (fingerprint authentication, iris and face identification, earlobe geometry identification), the gait recognition has the advantage of identifying persons with low resolution or hidden camera shots [3]. Physiological biometric methods cannot reliably detect non-cooperating individuals, especially not at distances, under the changing environmental conditions of the real world [4]. However, the accuracy of walking recognition can be greatly influenced by several factors, such as the viewing angle that changes the visual attributes [5].

\section{$3 \mathrm{Leg} /$ foot ratio analysis}

The essence of this analysis is that we determine the ratio of the leg's and foot's lengths with a program developed in LabVIEW. We first measured the ratio of one of our legs, which ranged between 2.42 and 2.43 , so we selected these two numbers as limit values. So, if the ratio of the leg and foot measured in pixels is between these two numbers, the program will give a green signal.

Fig. 1 shows a measurement in trousers and in slippers. The proportional value (2.26314) did not correspond to the set limits, so the green light did not flash. Fig. 2 shows another measurement. In this case, the person was sitting in socks, without any footwear. In this 
case, the proportional value was adequate, and the green light flashed. In shorts or without socks the green light also flashed. It can be stated that the clothes have minimal influence on the results, but the footwear has an important role in this measurement.

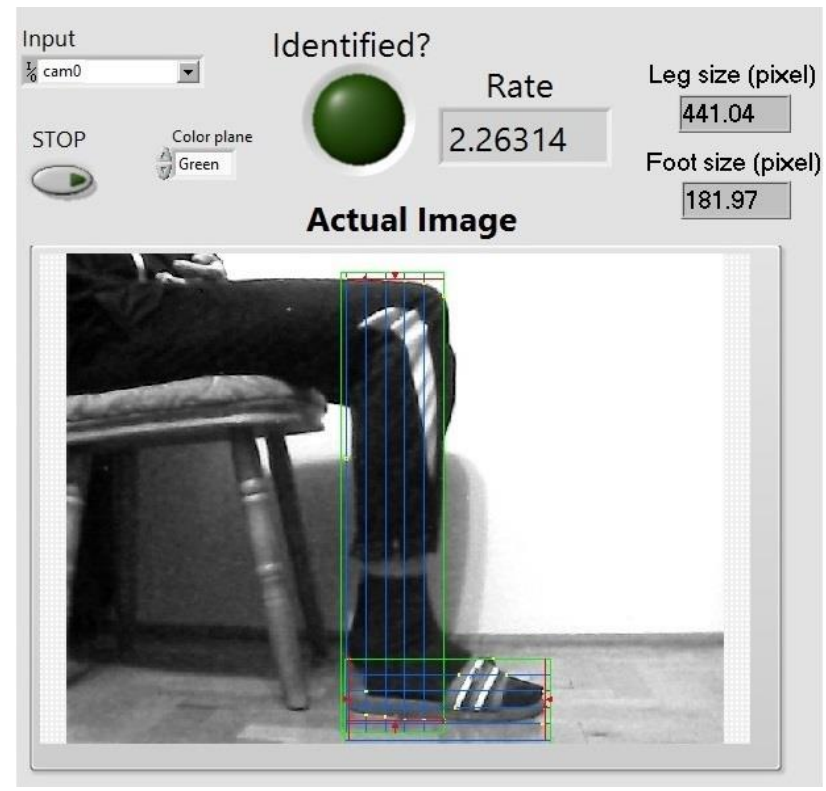

Fig. 1. Measuring in slippers.

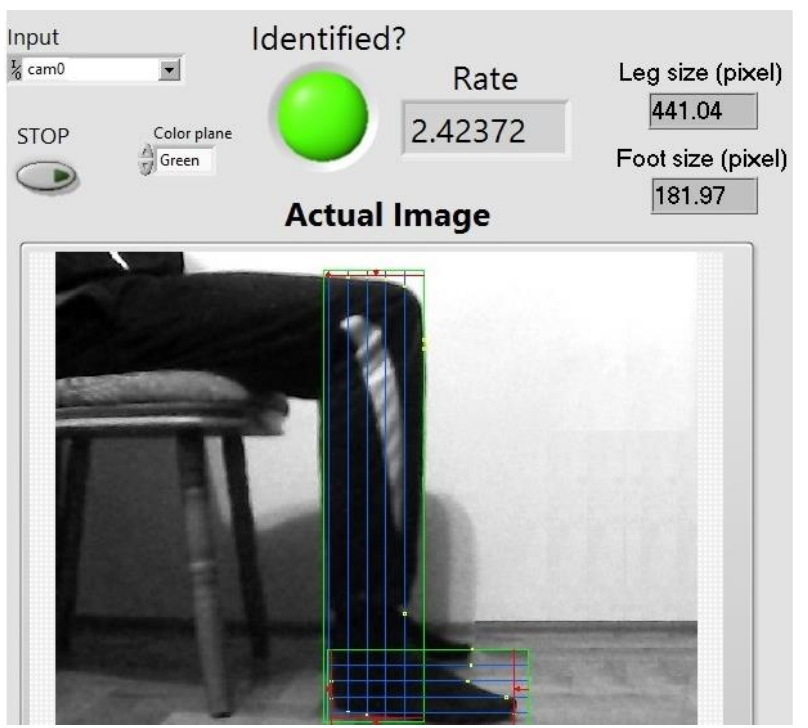

Fig. 2. Measuring without any footwear.

\section{The presentation of gait recognition software}

We attached a point to the knee and another to the leg of the person, about 35 centimetres from the ground. The background of the points is white, the point itself is black. It is important to have a light or white background to avoid false point detection, and to have your foot closer to the camera where the reference points are located. The measurements were made with artificial light, with normal room lighting, beside the darkened drapery avoiding sunlight, creating approximately the same lighting conditions for each measurement.

Fig. 3 shows the front panel while the program is working. On the right side of the figure, the coordinates of the detected points will be stored in blocks after running. X1 and Y1 are valid for the first point (suited on the knee), while $\mathrm{X} 2$ and $\mathrm{Y} 2$ are valid for the second point. In the middle it is the actual image, the two red rectangles frame the reference points. On the second and third tabs, the gait image is processed. On the second tab the actual gait image is processed, while on the third tab the saved and reloaded gait image is processed. If the actual gait image is like the saved one, the green light below the actual image will flash. The program suits a function on the curves drawn by the two reference points. You can choose between four function models (Linear, Polynomial, Exponential, Power) and you can choose the order of the function too.

On Fig. 4. a) and b) the blue points represent the detected points and the red curves are the functions suited on them. According to the tests, the most accurate fit is the fourth-degree (biquadratic) polynomial function (Fig 4. a)). However, for biquadratic polynomials, the third and fourth coefficients take very small values (up to $10^{-10}$ ), and the second-order (quadratic) function also follows well the curve drawn by the points (Fig 4. b)), so the use of the fourth-degree polynomials becomes unnecessary. Working with quadratic function is simpler than working with biquadratic ones.

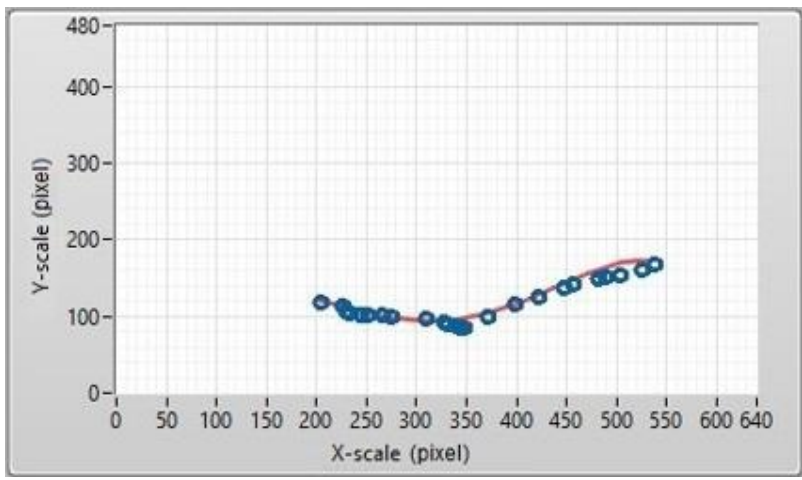

Fig. 4. a) Suiting a fourth-degree polynomial function on the gait curve.

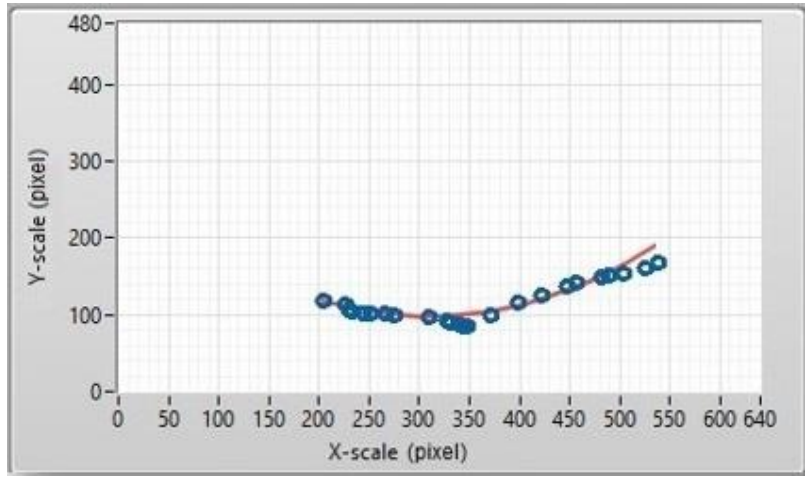

Fig. 4. b) Suiting a second-degree polynomial function on the gait curve. 


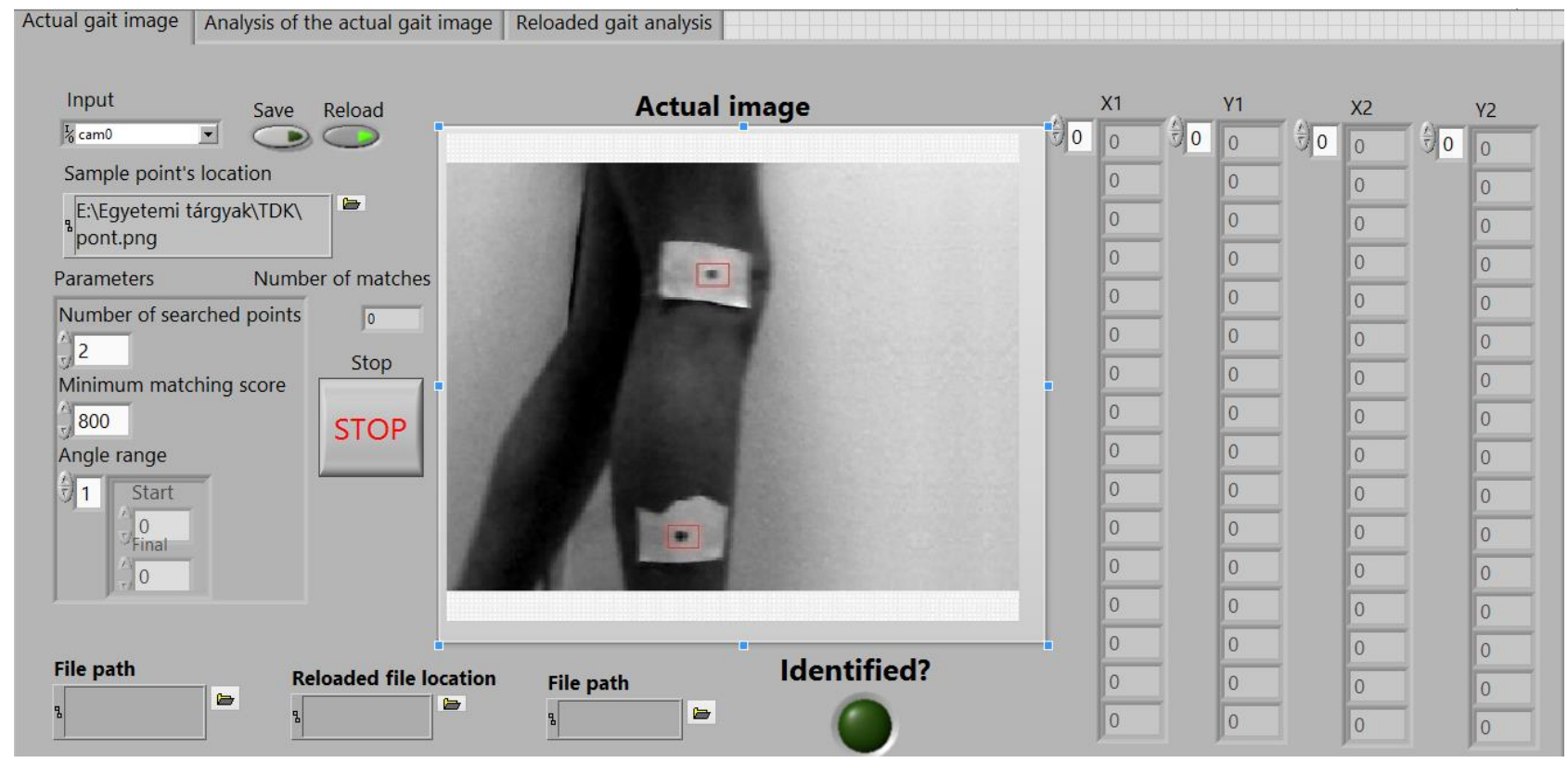

Fig. 3. The front panel of the program while working.

\section{Gait recognition results in different conditions}

Fig. 5. shows the coefficients of the functions suited on the curve drawn by the first (blue columns) and the second point (green columns). During this series of measurements, the tested person wore shorts and socks without any shoes or slippers, and the first step was made with the right leg (the reference points were also fixed on the right leg). According to the results, in 8 cases out of 10 the system recognized the tested person. The unsuccessful attempts are written with red characters.

\begin{tabular}{|c|c|c|c|c|c|}
\hline \multicolumn{3}{|c|}{ In shorts, 1 . point - no footwear } & \multicolumn{3}{|c|}{ In shorts, 2. point - no fw } \\
\hline$x^{\wedge} 0$ & $x^{\wedge} 1$ & $x^{\wedge} 2$ & $x^{\wedge} 0$ & $x^{\wedge} 1$ & $x^{\wedge} 2$ \\
\hline 399.7463 & -0.0469 & $-3.46 \mathrm{E}-06$ & 185.8495 & 0.5765 & 0.0011 \\
\hline 396.7941 & -0.0554 & 3.05E-05 & 213.5363 & -0.8573 & 017 \\
\hline 404.3768 & & & & & 1007 \\
\hline 400.0114 & -0.0578 & 3.05E-05 & 227. & -0.8 & 0.0016 \\
\hline 393.8913 & 0.0003 & $-9.64 E-05$ & 226.7635 & -0.9018 & 0.0017 \\
\hline 404.5176 & -0.1228 & $1.00 \mathrm{E}-04$ & 234.8857 & -0.9617 & 0.0018 \\
\hline 406.8849 & -0.1174 & & & -0.7867 & 0.0015 \\
\hline 390.3333 & 0.0225 & $-1.00 \mathrm{E}-04$ & 209.1956 & -0.7424 & 0.0013 \\
\hline 395.9785 & -0.0232 & & 220.0219 & -0.7801 & 0.0013 \\
\hline 397.4828 & -0.0574 & 3.27E-05 & 179.0767 & -0.546 & 0.0011 \\
\hline
\end{tabular}

Fig. 5. Results in shorts, no footwear.

On Fig. 6. you can see the coefficients of the functions received from measurements made in shorts wearing slippers. The results are very similar to the first measurement, the False Rejection Rate (FRR) increased a bit: the tested person was recognized 7 times out of 10 measurements.

\begin{tabular}{|c|c|c|c|c|c|}
\hline \multicolumn{3}{|c|}{ In slippers, 1. point } & \multicolumn{3}{|c|}{ In slippers, 2. point } \\
\hline$x^{\wedge} 0$ & $x^{\wedge} 1$ & $x^{\wedge} 2$ & $x^{\wedge} 0$ & $x^{\wedge} 1$ & $x^{\wedge} 2$ \\
\hline 413.5568 & -0.0701 & $6.55 \mathrm{E}-05$ & 217.3 & -0.7 & 0.0015 \\
\hline 405.3897 & -0.0107 & $-5.83 E-05$ & 225.0 & -0.7 & .0014 \\
\hline 417.8 & .093 & $7.29 \mathrm{E}-05$ & 262. & 75 & 0.0021 \\
\hline 413.9129 & -0.0565 & -05 & 1 & & 00 \\
\hline 414. & $-0 . \mathrm{C}-x+y-1)$ & $3.15 \mathrm{E}-05$ & 22 & -0 . & 0.0016 \\
\hline 423.2 & & $E-04$ & 4.1 & & 0.001 \\
\hline 412. & -0.0507 & 1.9 & 12 & -0 . & 012 \\
\hline & & -05 & 2 & & 0.0013 \\
\hline 424. & -0.15 & $E-04$ & -1 & & 0.0019 \\
\hline 415.4354 & -0.0592 & 3.53E-05 & 210.8684 & -0.6811 & 0.0014 \\
\hline
\end{tabular}

Fig. 6. Results in shorts, in slippers. The unsuccessful attempts are written with red characters.

Fig. 7. shows the data received from measurements made in trousers, without shoes. The FRR increased to $50 \%$, so only half of the measurements were successful. These results can be explained with the fact that the reference points fixed on the trousers were moving with the cloth (the trousers are moving on the leg while walking), while fixed directly on the tested person's knee and leg, they followed exactly the movement of the knee and the leg.

\begin{tabular}{|c|c|c|c|c|c|}
\hline \multicolumn{3}{|c|}{ In trousers, 1 . point - no fw } & \multicolumn{3}{|c|}{ In trousers, 2. point - no fw } \\
\hline$x^{\wedge} 0$ & $x^{\wedge} 1$ & $x^{\wedge} 2$ & $x^{\wedge} 0$ & $x^{\wedge} 1$ & $x^{\wedge} 2$ \\
\hline 401.4465 & -0.1794 & 0.0002 & 270.2624 & -1.285 & 0.0023 \\
\hline 389.2778 & -0.134 & 0.0002 & 152.8095 & -0.4528 & 0.0009 \\
\hline 384.0136 & -0.0881 & 0.0001 & 169.2744 & -0.7754 & 0.0018 \\
\hline 377.0618 & -0.0694 & 0.0001 & 161.3756 & -0.6282 & 0.0015 \\
\hline 382.4782 & -0.0582 & 0.0001 & 198.1475 & -0.8582 & 0.0016 \\
\hline 401.3688 & -0.2588 & 0.0004 & 256.0288 & -1.4425 & 0.0032 \\
\hline 381.9576 & -0.1289 & 0.0002 & 167.4292 & -0.6569 & 0.0014 \\
\hline 385.261 & -0.2386 & 0.0002 & 203.0616 & -0.9265 & 0.0019 \\
\hline 373.701 & -0.0971 & 0.0002 & 174.136 & -0.7995 & 0.002 \\
\hline 375.3779 & -0.1076 & 0.0002 & 171.3232 & -0.8775 & 0.0024 \\
\hline
\end{tabular}

Fig. 7. Results in trousers, no footwear. The unsuccessful attempts are written with red characters. 
Fig. 8. shows the results from a series of measurements made in shorts, wearing no shoes, the reference points fixed on the left leg, and going from the right of the camera to the left. The previous measurements were made from the left of the camera to the right. With these attempts, the FRR became $90 \%$, only one time out of 10 was the test person recognized. This result was caused by the fact that the software was calibrated to start from the left of the camera. The curves drawn by the reference points fixed on the left or on the right leg are different.

\begin{tabular}{|rlrcccc}
\hline \multicolumn{2}{c}{ Opposite direction -1 point } & \multicolumn{4}{c}{ Opposite direction - 2. point } \\
\hline$X^{\wedge} 0$ & $X^{\wedge} 1$ & \multicolumn{1}{c}{$X^{\wedge} 2$} & \multicolumn{1}{c}{$X^{\wedge} 0$} & \multicolumn{1}{c}{$X^{\wedge} 1$} & $X^{\wedge} 2$ \\
343.794 & 0.2355 & -0.0002 & 215.5685 & -0.8344 & 0.0013 \\
326.8067 & 0.3227 & -0.0003 & 234.3298 & 0.9133 & 0.0014 \\
365.491 & 0.1453 & -0.0002 & 225.2881 & -0.8438 & 0.0013 \\
328.3563 & 0.3319 & -0.0004 & 283.1634 & -1.2319 & 0.0019 \\
338.6368 & 0.2772 & -0.0003 & 211.0794 & -0.7592 & 0.0012 \\
365.3066 & 0.1254 & -0.0001 & 265.4828 & -1.1291 & 0.0019 \\
342.1156 & 0.2323 & -0.0002 & 193.6888 & -0.6821 & 0.0011 \\
368.3189 & 0.1154 & -0.0001 & 214.2421 & -0.7694 & 0.0011 \\
388.4625 & 0.0199 & $2.85 E-05$ & 225.5323 & -0.949 & 0.0016 \\
353.3414 & 0.2108 & -0.0002 & 253.5832 & -1.0858 & 0.0017
\end{tabular}

Fig. 8. Results in shorts, walking in the opposite direction. The unsuccessful attempts are written with red characters.

The measurements presented above were made with starting the walking with the right leg. We have made some measurements with the same conditions as in the first measurement series (reference points on right leg, wearing short and no footwear), but the first step was made with the left leg. The results are shown on Fig. 9. Because the coefficients were very different from the initial values, we made only three attempts, certainly without any success. The curves drawn by the two reference points, that are shown on Fig. 10. a) and b) were far from the initial curves because of the cyclical shift and because the camera can view only a few steps. So, it is normal that the program didn't make any recognition in this measurement.

\begin{tabular}{|ccr|rll|}
\hline \multicolumn{2}{c}{ Starting with left - 1. point } & \multicolumn{3}{c|}{ Starting with left - 2. point } \\
\hline$X^{\wedge} 0$ & $X^{\wedge} 1$ & \multicolumn{1}{c|}{$X^{\wedge} 2$} & \multicolumn{1}{c|}{$X^{\wedge} 0$} & \multicolumn{1}{c|}{$X^{\wedge} 1$} & \multicolumn{1}{c|}{$X^{\wedge} 2$} \\
358.0921 & 0.1561 & -0.0001 & 150.8065 & 0.2241 & -0.0005 \\
355.9683 & 0.0869 & $-1.36 E-05$ & 150.1471 & 0.1522 & -0.0004 \\
323.1626 & 0.259 & -0.0002 & 164.19 & 0.1417 & -0.0004 \\
\hline
\end{tabular}

Fig. 9. Unsuccessful results starting with the left leg.

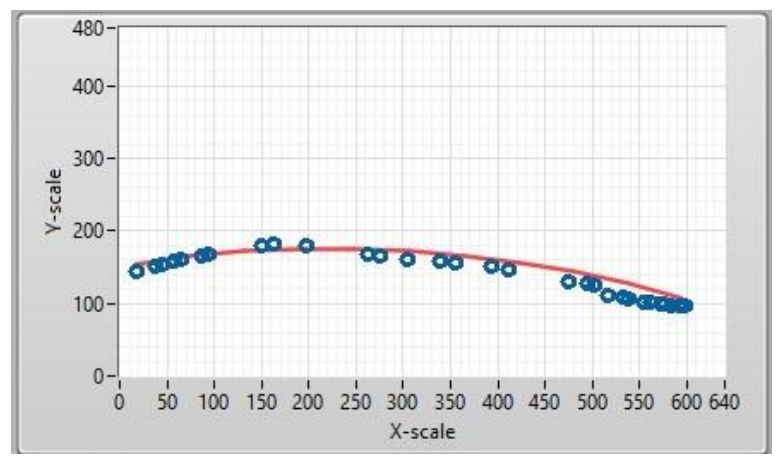

Fig. 10. a). Curve drawn by the second reference point starting the walking with left leg

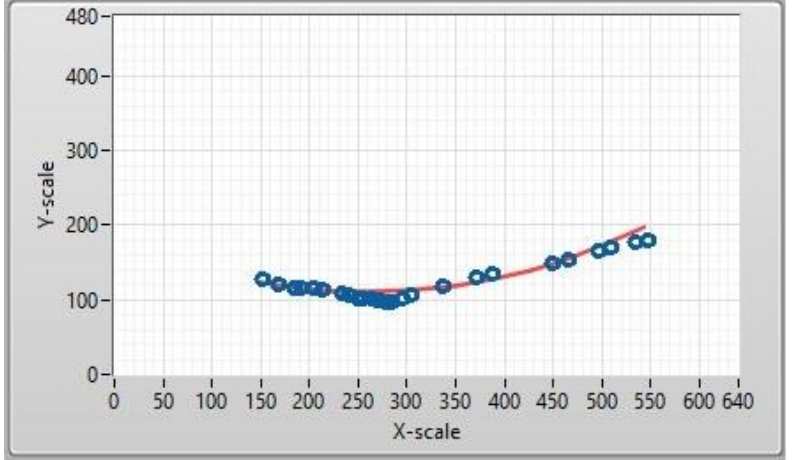

Fig. 10. b). Curve drawn by the second reference point starting the walking with right leg

\section{Conclusion}

With these tests, we have showed how our gait recognition system is working in different situations. According to the result, the footwear has the least influence on the system, but wearing trousers, or walking in the opposite direction can have serious impact on the successfulness of the test. It is also important to start the measurement with the right leg, because otherwise the curves will be far different from the reference curves, and the program will not recognize even the person with permission.

\section{Acknowledgement}

The work/publication is supported by the EFOP-3.6.1-162016-00022 project. The project is co-financed by the European Union and the European Social Fund.

\section{References}

1. Jeffrey E. Boyd, James J. Little: Biometric Gait Recognition, 19-42 (Springer - Verlag Berlin Heidelberg, 2005)

2. Lee, Lily, W. Eric L. Grimson. Gait analysis for recognition and classification. Automatic Face and Gesture Recognition, 2002. Proceedings. Fifth IEEE International Conference on (pp. 155-162). IEEE, 2002.

3. Mark S. Nixon, T. N. Tan, R. Chellappa: Human Identification Based on Gait, (Springer Science + Business Media, Inc., 2006)

4. Ju Han, Bir Bhanu: Individual Recognition Using Gate Energy Image, 28.2: 316-322, (IEEE transactions on pattern analysis and machine intelligence, 2006)

5. Wei Zeng, Cong Wang: Neurocomputing - Viewinvariant gait recognition via deterministic learning, 175: 324-335 (2016) 